\title{
Analysis and Experimental Evaluation of Rate Adaptation with Transmit Buffer Information
}

\author{
Yongjiu Du, Dinesh Rajan, and Joseph Camp \\ Department of Electrical Engineering, Southern Methodist University (SMU), Dallas, TX \\ \{ydu, rajand, camp $\} @$ smu.edu
}

\begin{abstract}
In hardware, packet loss may happen due to overflow from a finite-depth transmit buffer. To prevent such losses and further improve rate selection, we exploit statistical knowledge of transmit buffer occupancy and source packet distribution in IEEE 802.11-based systems, which have variable frame slots. We consider a traditional method of rate adaptation based on channel quality information and evaluate the throughput gain in hardware when the buffer occupancy and source packet distribution information are known. Our optimization objective is to maximize the throughput with constant transmit power since most IEEE 802.11 APs and nodes operate in this manner. We also derive an upper bound of the improvement introduced by exploiting the offered load distribution and buffer status information. By evaluating the effect of diverse buffer sizes with different packet arrival distributions, both our theoretical analysis and our experimental results show that the throughput can be greatly improved in many cases when the source packet distribution and buffer status information are exploited.
\end{abstract}

Keywords-Rate Adaptation; Transmit Buffer Information; Markov Chain; SNR; FPGA; Implementation;

\section{INTRODUCTION}

Rate adaptation is widely used to increase spectrum efficiency in the time-varying wireless channels. Packet loss/success-based rate adaptation protocols have been well studied and widely implemented in the past decade [1]-[5]. This kind of protocol uses packet loss/success statistics to select the optimal rate to transmit data packets. However, packet-level information is coarse-grained and usually takes tens of transmissions to get a reasonable estimate of the channel quality. As a result, its performance is known to degrade as the Doppler effect increases. To solve the rate adaptation with high mobility, a variety of channel-based rate adaptation protocols have been developed [6]-[9]. SNR-based protocols can adapt to fast-fading channels. However, the SNR is not always an accurate indicator of PER for OFDM systems in various frequency-selective channels. To address this, soft information from SISO (Soft In Soft Out) decoders has been used to determine the best rate, which has much better performance in multi-path channels [8]. Additional improvements have come from a novel effective SNR metric for rate adaptation, achieving better performance than protocols that are solely based on SNR [9]. These SNR-based schemes have yet to be widely used in commercial systems.

Traditional rate adaptation protocols usually assume a fully backlogged transmit buffer. However, in real hardware, the

This research was supported by NSF grants CNS-0958436, CNS-1040429, and CNS-1150215. buffer depth is always finite. There could be a full or empty status for the buffer. The packet loss in a system without retransmissions results from either packet overflow in the transmit buffer or packet corruption in the channel. Consequently, using both the buffer information and channel information for rate adaptation may achieve superior performance [10]-[14]. These works use either statistical or instantaneous information of the buffer occupancy and channel quality to adaptively change the transmission rate, which allows significant performance gain. However, all works above leverage simulation to illustrate the importance of the buffer status information without implementation in hardware. Moreover, each work assumes a constant frame slot, within which different number of packets are sent for different rates. They aim to minimize the packet loss rate given the constant frame duration $T$, the channel model $h(t)$, the constant buffer size $M$, and the offered load distribution $P_{\lambda}$. However, many protocols use variable frame slots (e.g., IEEE 802.11, HIPERLAN/2, IEEE 802.15.3, ZigBee and IEEE 802.16), which have been widely-used in commercial applications. In these systems, only one packet is transmitted in one frame slot. As a result, the frame duration in these protocols varies for different packet sizes and transmission rates. In this system model, we optimize the throughput given the constant packet size $L$, the channel model $h(t)$, the constant buffer size $M$, and the offered load distribution $P_{\lambda}$. Therefore, we are not able to apply the optimization model of constant-frame-slot systems to variableframe-slot systems. We will discuss such a mechanism based on the IEEE 802.11 PHY standard in this paper. Moreover, for many devices and low cost transceivers, packet-level power adaptation is not available. Thus, we only discuss constant transmit power rate adaptation in this work.

In this paper, we analyze the performance improvement by exploiting the offered load distribution and buffer status information. In order to get the optimal rate adaptation thresholds, we leverage a steady-state analysis of a joint buffer and channel quality Markov Chain. Furthermore, we derive an upper bound of the throughput improvement by exploiting the offered load distribution and buffer status information. Based on our theoretical analysis, we experimentally evaluate the proposed rate adaptation mechanism on an FPGA-based hardware platform.

The main contributions of our work are as follows:

1) Formulate and analyze a rate adaptation system based on the IEEE 802.11 standard, and present a rate adaptation solution for this variable-frame-slot model.

2) Derive an upper bound of the improvement by exploiting offered load distribution and the buffer in- 


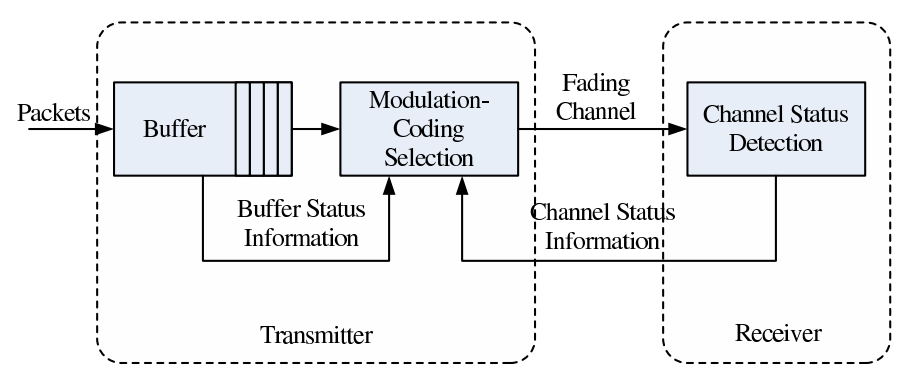

Fig. 1. Rate adaptation with offered load distribution and buffer status provided

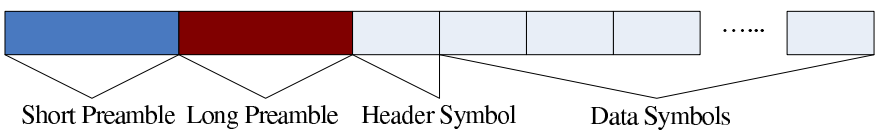

Fig. 2. IEEE 802.11 PHY frame Structure, which is used in the analysis and evaluation in this work

\section{formation.}

3) Experimentally evaluate the throughput performance and empirically verified the theoretical analysis on a diverse set of wireless channels.

The paper is organized as follows. Section II presents a system model based on the IEEE 802.11 PHY standard and theoretically analyzes the methodology to choose the rate adaptation parameters to optimize the throughput. Next, an upper bound is analyzed and derived for the throughput improvement by exploiting offered load distribution and buffer status information in Section III. An experimental evaluation system and numerical results are provided in Section IV which show much better performance than single layer rate adaptation systems. Related work is presented in Section V. Finally, in Section VI, some concluding remarks and suggestions for future research are presented.

\section{System Model}

A typical model for rate adaptation contains a transmitter, a receiver, a forward channel and a feedback channel, as shown in Figure 1. The packet comes from the higher layers into the transmit buffer according to a certain random process (e.g., a Poisson or Bernoulli distribution). The transmitter picks a packet from the buffer and sends it over the channel with one of the transmit rates in each frame slot. The receiver demodulates and decodes the received signal and also estimates and sends the channel information back to the transmitter through the feedback channel.

In this paper, we use constant transmit power, although there are several works that consider power adaptation [12], [15], [16]. Our assumption is that many low-cost transmitters usually use the default power settings and do not change the transmit power at the packet level.

\section{A. Dynamic Transmit Rate}

We use a frame structure as described in the IEEE 802.11 standard. One frame is composed of a short preamble, a long preamble, a header symbol and several data symbols [17], as shown in Figure 2. Both the short preamble and the long preamble have a duration of two OFDM symbols. The header
TABLE I. TRANSMISSION RATE PARAMETERS

\begin{tabular}{|c|c|c|c|}
\hline Rate Index & Constellation & Code Rate & $R_{n}$ (bit) \\
\hline 0 & BPSK & $1 / 2$ & 24 \\
\hline 1 & BPSK & $3 / 4$ & 36 \\
\hline 2 & QPSK & $1 / 2$ & 48 \\
\hline 3 & QPSK & $3 / 4$ & 72 \\
\hline 4 & 16QAM & $1 / 2$ & 96 \\
\hline 5 & 16QAM & $3 / 4$ & 144 \\
\hline 6 & 64QAM & $2 / 3$ & 192 \\
\hline 7 & 64QAM & $3 / 4$ & 216 \\
\hline
\end{tabular}

TABLE II. PER APPROXIMATION PARAMETERS FOR EACH RATE

\begin{tabular}{|c|c|c|c|c|c|c|c|c|}
\hline Rate & 0 & 1 & 2 & 3 & 4 & 5 & 6 & 7 \\
\hline$a_{n}$ & 1.2 & 4 & 6 & 8 & 20 & 20 & 18 & 6 \\
\hline$g_{n}$ & 1.8 & 1.2 & 1.3 & 2 & 2.8 & 7 & 20 & 50 \\
\hline
\end{tabular}

has a duration of one symbol. We assume the packet is $L$ bytes. In the data symbols, there are 16-bit service data and six convolutional code tail bits. Let $R_{n}$ denote the number of data bits that can be transmitted in one OFDM symbol in rate $n$, as shown in Table I [17]. The transmitter sends one packet every frame slot. The packet length could be from 1 to 2047 bytes. The number of symbols in a frame is:

$$
N_{s}(n, L)=\left\lceil 5+\frac{(8 L+16+6)}{R_{n}}\right\rceil
$$

Let $T_{s}$ denote the duration of one OFDM symbol. In this paper, we assume a constant payload size. Moreover, if there are no packets in the buffer, the transmitter still sends probe frames that only include the preamble and header symbol to enable the receiver to continue to measure the channel quality. The frame slot duration is:

$T_{f}(m, n)=T_{f}(m, n, L)= \begin{cases}5 T_{s}+T_{d} & \text { if } \mathrm{m}=0 \\ T_{s} N_{s}(n, L)+T_{d} & \text { otherwise }\end{cases}$

where $T_{d}$ is a fixed-time delay including demodulation, decoding, and feedback. The number of packets in the buffer is $m$.

The variation of PER with SNR for rate $n$ is denoted by $P E R_{n}(\gamma)$. Since it is challenging to get a closed form expression of $P E R_{n}$ in a coded system, we use the following approximation from [14] to denote the PER as:

$$
P E R_{n}(\gamma)=\min \left(1, a_{n} \exp \left(-\gamma / g_{n}\right)\right)
$$

where $a_{n}$ and $g_{n}$ are the parameters to describe $P E R$ for rate $n$, and $\gamma$ is the SNR value. The parameters for packet length $L=1024$ bytes are shown in Table II.

\section{B. Diverse Offered Load}

In general, we assume that the packets arrive randomly at the buffer. For example, packets from wide-area networks arrive according to a Poisson- or Bernoulli-distributed process [18]. In this paper, we model the arrivals as a Poisson process with an average packet arrival rate of $\lambda$ packets per second. In a time interval of $t$, the probability of $k$ packets arriving is given by [19]:

$$
p_{k}=\frac{(\lambda t)^{k}}{k !} \exp (-\lambda t) \quad k=0,1,2 \ldots
$$


Packets arriving at the buffer during one packet transmission follow a Poisson distribution with an expectation of $\lambda T_{f}(m, n)$. The distribution is given by:

$$
p_{k}=\frac{\left(\lambda T_{f}(m, n)\right)^{k}}{k !} \exp \left(-\lambda T_{f}(m, n)\right)
$$

\section{Dynamic Channel Quality}

For the wireless channel, a Rayleigh fading model is a good approximation and agrees well with empirical observations for mobile wireless links [20]. Let $\gamma$ denote the received SNR. The distribution of $\gamma$ can be expressed as [20]:

$$
p_{\gamma}=\frac{1}{\bar{\gamma}} \exp \left(-\frac{\gamma}{\bar{\gamma}}\right)
$$

where $\bar{\gamma}$ is the expected value of SNR.

We divide the whole SNR region into $N$ non-overlapping regions. The number of feasible rates in which packets can be transmitted is also $N$. We define the thresholds as $\gamma_{0}=$ $0<\gamma_{1} \cdots<\gamma_{N}=\infty$. If the instantaneous SNR falls into the region between $\gamma_{n}$ and $\gamma_{n+1}$, we say the channel is in state $n$ and we use rate $n$ to transmit.

For simplicity, we assume the channel is block-fading. The channel keeps the current state or changes to the adjacent states according to the following cross-rate probability, which is suitable for slow-fading wireless channels [21], [22]:

$$
\begin{gathered}
p_{n, n+1}=\frac{N_{n+1} T_{f}(m, n)}{p_{\gamma_{n}}} \quad \text { if } n=0, \ldots, N-2 \\
p_{n, n-1}=\frac{N_{n} T_{f}(m, n)}{p_{\gamma_{n}}} \quad \text { if } n=1, \ldots, N-1
\end{gathered}
$$

where $T_{f}$ is the ongoing time slot. $N_{n}$ is denoted as [22]:

$$
N_{n}=\sqrt{\frac{2 \pi \gamma_{n}}{\bar{\gamma}}} f_{d} \exp \left(-\frac{\gamma_{n}}{\bar{\gamma}}\right)
$$

where the Doppler shift $f_{d}=v / \lambda^{\prime}$ and represents the ratio of the relative velocity between the transmitter and the receiver and the carrier wavelength.

Assume $p_{\gamma_{n}}$ is the probability that the channel quality falls into the region $\left[\gamma_{n}, \gamma_{n+1}\right)$. We can calculate $p_{\gamma_{n}}$ from (6):

$$
p_{\gamma_{n}}=\int_{\gamma_{n}}^{\gamma_{n+1}} p_{\gamma} d \gamma
$$

\section{Steady State Analysis}

We model the buffer state transition as a queue service process. If we assume that the buffer is able to accommodate $M$ packets, the buffer state $C_{i} \in\{0,1, \ldots, M\}$. Let $R_{i}$ denote the rate for the $i t h$ transmission. We assume $\left(C_{i}, R_{i}\right)$ is the joint buffer and channel state. We then define a transition matrix $P$, as in (12), where the element $p_{(m, n) \rightarrow(s, t)}$ denotes the transition probability from the state $\left(C_{i}=m, R_{i}=n\right)$ to state $\left(C_{i+1}=s, R_{i+1}=t\right)$.
Let $\pi_{i, j}$ denote the probability of buffer state $i$ and channel state $j$, and define the row vector $\pi$ as:

$$
\pi=\left[\pi_{0,0}, \ldots, \pi_{0, N-1}, \ldots, \pi_{M, N-1}\right]
$$

For the steady state of this system, we have

$$
\pi=\pi P
$$

and

$$
\sum_{0 \leq i \leq M, 0 \leq j \leq N-1} \pi_{i, j}=1
$$

If the buffer is empty, the transmitter will send probe packets. Otherwise, the transmitter will send a packet at the rate corresponding to the current channel state. Hence the probability distribution of the next channel state depends on both the current channel state and the current buffer status. Also, the buffer state transition depends both on the offered load distribution and the current channel state. We have:

$$
p_{(m, n) \rightarrow(s, t)}=p(m \rightarrow s \mid n) p(n \rightarrow t \mid m)
$$

We first discuss the buffer state transition probability $p(m \rightarrow s \mid n)$. If the buffer is empty, the next state can be any state from 0 to $M$, and the buffer state transition only depends on the packet arrival process, as described by (5). If the incoming packets exceed $\mathrm{M}$, all the following packets will be dropped due to overflow. However, if there is at least one packet in the buffer, there will be one packet transmitted when a new transmission starts. As a result, the next state can be any state from $m-1$ to $M$. Since the system can transmit at most one data packet in one frame, there is a constraint of $s-m \geq-1$. The transition probability of the buffer states is:

$p(m \rightarrow s \mid n)= \begin{cases}p_{k=0} & \text { if } m=s=0 \text { or } s-m=-1 \\ p_{k \geq s-m+1} & \text { else if } s=M \\ p_{k=s-m+1} & \text { else if } s-m \geq 0\end{cases}$

According to the input packet distribution, we have

$p_{k \geq x}=\sum_{x \leq k \leq \infty} p_{k}=\sum_{x \leq k \leq \infty} \frac{\left(\lambda T_{f}(m, n)\right)^{k}}{k !} \exp \left(-\lambda T_{f}(m, n)\right)$

For the channel state transition, if the channel is in state 0 , it can go to state 1 or stay in the current state. Similarly, if the channel state is $N-1$, it can go to state $N-2$ or stay in the current state. For simplicity, we assume that the channel can only stay in the current state or change to the adjacent states in other cases. We have the following transition probability:

$$
\begin{aligned}
& p(n \rightarrow t \mid m)= \\
& \begin{cases}1-p_{0 \rightarrow 1} & \text { if } n=t=0 \\
1-p_{N-1 \rightarrow N-2} & \text { elsif } n=t=N-1 \\
1-p_{n \rightarrow n-1}-p_{n \rightarrow n+1} & \text { elsif } n=t \\
p_{n \rightarrow n-1} & \text { elsif } t=n-1 \\
p_{n \rightarrow n+1} & \text { elsif } t=n+1 \\
0 & \text { elsif }|t-n|>1\end{cases}
\end{aligned}
$$

Now, we can solve (14) and obtain the steady-state distribution. Our objective is to minimize the total packet loss due 


$$
P=\left[\begin{array}{cccccc}
p_{(0,0) \rightarrow(0,0)} & \cdots & p_{(0,0) \rightarrow(0, N-1)} & p_{(0,0) \rightarrow(1,0)} & \cdots & p_{(0,0) \rightarrow(M, N-1)} \\
p_{(0,1) \rightarrow(0,0)} & \cdots & p_{(0,1) \rightarrow(0, N-1)} & p_{(0,1) \rightarrow(1,0)} & \cdots & p_{(0,1) \rightarrow(M, N-1)} \\
\vdots & \ddots & \vdots & \vdots & \ddots & \vdots \\
p_{(0, N-1) \rightarrow(0,0)} & \cdots & p_{(0, N-1) \rightarrow(0, N-1)} & p_{(0, N-1) \rightarrow(1,0)} & \cdots & p_{(0, N-1) \rightarrow(M, N-1)} \\
p_{(1,0) \rightarrow(0,0)} & \cdots & p_{(1,0) \rightarrow(0, N-1)} & p_{(1,0) \rightarrow(1,0)} & \cdots & p_{(1,0) \rightarrow(M, N-1)} \\
\vdots & \ddots & \vdots & \vdots & \ddots & \vdots \\
p_{(M, N-1) \rightarrow(0,0)} & \cdots & p_{(M, N-1) \rightarrow(0, N-1)} & p_{(M, N-1) \rightarrow(1,0)} & \cdots & p_{(M, N-1) \rightarrow(M, N-1)}
\end{array}\right]
$$

to both buffer overflow and channel corruption. The packet loss due to buffer overflow is:

$$
p_{\text {overflow }}(m, n)=\sum_{v<k \leq \infty} k p_{k}
$$

where $v$ is the available space in the buffer and can be described as:

$$
v= \begin{cases}M & \text { if } m=0 \\ M-m+1 & \text { otherwise }\end{cases}
$$

Assume $p_{f}(n)$ is the average PER of the transmission in channel state $n$, which can be expressed as:

$$
p_{f}(n)=\int_{\gamma_{n}}^{\gamma_{n+1}} P E R_{n}(\gamma) p_{\gamma} d \gamma
$$

The packet loss objective function could be described as:

$$
P_{\text {loss }}=\sum_{\substack{0 \leq m \leq M, 0 \leq n \leq N-1 \\ \sum_{1 \leq m \leq M, 0 \leq n \leq N-1}} p_{f}(n) q_{m, n}} p_{\text {overflow }}(m, n) q_{m, n}+
$$

where $q_{m, n}$ is the average number of slots in state $(m, n)$ per second. When the buffer is empty, we only send probe packets. As a result, there is only packets overflow probability, without packet corruption in the channel. In other states, packets suffer from both buffer overflow and channel corruption.

$$
q_{m, n}=\frac{\pi_{m, n}}{\sum_{0 \leq m \leq M, 0 \leq n \leq N-1} \pi_{m, n} T_{f}(m, n)}
$$

We seek to find the optimum thresholds $\gamma_{1}, \ldots, \gamma_{N-1}$ to minimize the total packet loss:

$$
\underset{\gamma_{1}, \ldots, \gamma_{N-1}}{\arg \min } P_{l o s s}\left(\gamma_{1}, \ldots, \gamma_{N-1}\right)
$$

For this kind of optimization, we may rely on linear programming tools such as AMPL or MATLAB to achieve the best solution. In this work, however, we solve the optimization problem with an FPGA-based platform and repeatable, controlled channels to directly compare different SNR thresholds and buffer occupancies empirically.

\section{UPPER BOUND IMPROVEMENT OF EXPLOITING BUFFER INFORMATION}

\section{A. The Threshold Analysis}

Assuming $G_{n}$ is the number of packets transmitted per second in a rate adaptation system, we can express the instantaneous throughput $S_{n}(\gamma)$ when using rate $n$ as:

$$
S_{n}(\gamma)=G_{n}\left(1-P E R_{n}(\gamma)\right)
$$

Then, $\gamma_{n}$, which is the threshold between rate $n$ and rate $n-1$, can be solved by setting $S_{n-1}(\gamma)=S_{n}(\gamma)$ :

$$
G_{n-1}\left(1-P E R_{n-1}(\gamma)\right)=G_{n}\left(1-P E R_{n}(\gamma)\right)
$$

For rate adaptation with no offered load distribution and buffer status information, we always assume a full buffer status. Then, we can calculate $G_{n}$ as:

$$
G_{n}=\frac{1}{T_{f}(n)}
$$

where $T_{f(n)}$ is the transmission time slot for rate $n$.

Consequently, we can transform (27) to:

$$
\frac{1-P E R_{n-1}(\gamma)}{T_{f}(n-1)}=\frac{1-P E R_{n}(\gamma)}{T_{f}(n)}
$$

With fully backlogged nodes, this is the way to calculate the thresholds with the packet size and the PER information provided [13]. However, if the offered load is low, there will not always be backlogged packets in the buffer. When the offered load is far less than the channel capacity, there will be very few packets dropped due to overflow even if we always use the lowest rate to transmit. Consequently, $\gamma_{n}$ approaches $\infty$. We can conclude that, with offered load distribution and buffer status information, the optimal threshold for rate adaptation tends to move higher compared to that for rate adaptation with no offered load distribution and buffer status information.

\section{B. The Upper Bound Analysis}

As discussed above, we assume a full buffer when selecting the best threshold for a non-buffer-assisted rate adaptation system. When including offered load and buffer information, we will adjust the rate selection thresholds, which will result in decreasing the risk of channel corruption if there is a light packet load in the buffer. However, by doing this, the risk of buffer overflow will be increased. Assume $\overline{p_{O}}$ is the system packet overflow rate, and $\overline{p_{e}}$ is the packet error rate caused by 
the wireless channel. Both of them are for a system without considering the offered load distribution and the buffer status information. We denote $\overline{p_{o}}{ }^{\prime}$ and ${\overline{p_{e}}}^{\prime}$ as the counterparts of $\overline{p_{o}}$ and $\overline{p_{e}}$ when offered load distribution and buffer status information are involved. Compared to the throughput with non-buffer-assisted rate adaptation, systems with offered load distribution and buffer status information can achieve a normalized improvement of:

$$
\xi=\frac{\left(1-\overline{p_{o}}\right)\left(1-\overline{p_{e}}\right)-\left(1-\overline{p_{o}}\right)\left(1-\overline{p_{e}}\right)}{\left(1-\overline{p_{o}}\right)\left(1-\overline{p_{e}}\right)}
$$

If there is a low offered load, we will adjust the rate adaptation threshold higher to decrease the risk of channel corruption. However, risk of overflow in the buffer will correspondingly increase. As a result, we have $\overline{p_{o}}{ }^{\prime}>\overline{p_{o}}$ and ${\overline{p_{e}}}^{\prime}<\overline{p_{e}}$. Consequently, we have

$$
\begin{aligned}
\xi & =\frac{\left(1-\overline{p_{o}}\right)\left(1-\overline{p_{e}}\right)-\left(1-\overline{p_{o}}\right)\left(1-\overline{p_{e}}\right)}{\left(1-\overline{p_{o}}\right)\left(1-\overline{p_{e}}\right)} \\
& \leq \frac{\left(1-\overline{p_{o}}\right)\left(1-\overline{p_{e}}\right)-\left(1-\overline{p_{o}}\right)\left(1-\overline{p_{e}}\right)}{\left(1-\overline{p_{o}}\right)\left(1-\overline{p_{e}}\right)} \\
& =\frac{\overline{p_{e}}-\overline{p_{e}}}{1-\overline{p_{e}}} \\
& \leq \frac{\overline{p_{e}}}{1-\overline{p_{e}}} \\
& =I
\end{aligned}
$$

where $I$ is the possible improvement that can be achieved by possibly removing the packets error in the channel.

Thus, we can use $I$ to represent the upper bound of the throughput improvement by exploiting the offered load distribution and buffer status information. We can calculate $I$ by first calculating $\bar{p}_{e}$, where $\bar{p}_{e}$ is the average packet error rate and can be described as follows:

$$
\overline{p_{e}}=\sum_{n=1 \ldots N-1} \int_{\gamma_{n}}^{\gamma_{n+1}} p_{\gamma} P E R_{n}(\gamma) d \gamma
$$

Note that the packet errors for rate 0 can not be removed when we adjust the rate adaptation thresholds.

By applying (32) to (31), we can calculate the proposed upper bound for the throughput improvement by exploiting the offered load distribution and buffer status information.

\section{NUMERICAL ANALYSIS}

\section{A. Experiment Settings}

The FPGA-based platform we use for our experimental evaluation is the Wireless Open-Access Research Platform (WARP). WARP is a useful platform supporting a fully customized, cross-layer design [13]. Mainly, the PHY layer is implemented in the FPGA fabric, and the higher layers exist as $C$ code on an embedded PowerPC [23]. In contrast to the commonly-used reference design for WARP which heavily leverages Xilinx System Generator for the physical layer implementation, we use Verilog HDL to design and implement a full OFDM transceiver according to the IEEE 802.11 standard. We implement a complete real-time signal processing,

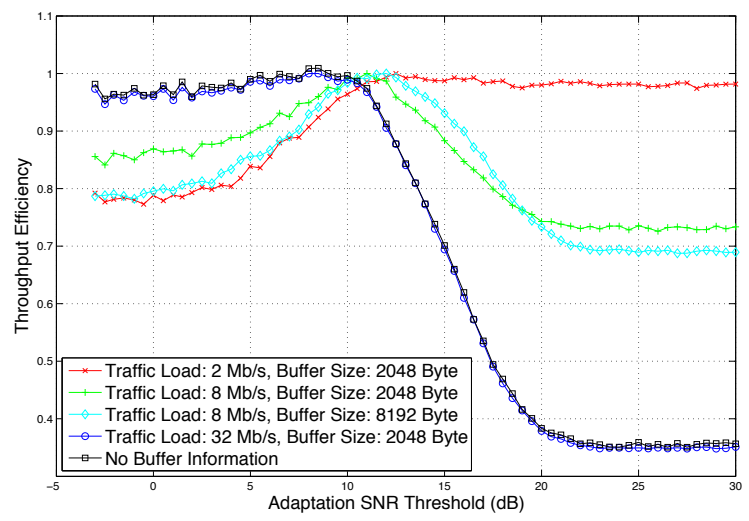

Fig. 3. Throughput efficiency with different SNR thresholds with a 2 packet buffer. Throughput efficiency is defined as the throughput with a certain threshold normalized by the throughput with the optimal threshold.

synchronization, and control system in the fabric of the FPGA. The PowerPC hosts the media access layer implementation in this system. We use the Azimuth ACE-MX channel emulator to generate channel effects, which provides approximately the same effects as complex over-the-air channels [24].

In our evaluation, we set the packet length $L=1024$ bytes. Note that, The optimal parameters for other packet lengths can be easily obtained by following the process in Section II. In order to cover all the SNR regions for all 8 rates, we set up a Rayleigh fading channel with an average SNR $\bar{\gamma}=31.5$, which corresponds to $15.0 \mathrm{~dB}$. Moreover, we set a Doppler shift of $25 \mathrm{~Hz}$. We generate the distribution of a packet source according to Poisson random process with three different average packet rates, $\lambda \in\{244,977,3906\}$ packets per second, corresponding to $\{2.0,8.0,32.0\} \mathrm{Mbps}$, respectively. We set the buffer size to 2 and 8 packets (2048 bytes and 8192 bytes), respectively, to examine the effect of different buffer sizes.

\section{B. Experimental Evaluation of Rate Adaptation}

Effect of Diverse Offered Load. To clearly demonstrate the effect of diverse offered load and buffer sizes, we now dynamically select between rate 0 and rate 4 (refer to Table I) according to the channel quality. From Figure 3, we can see that, for different offered loads, the optimal rate adaptation threshold varies. For a 32-Mbps stream, the best SNR threshold is approximately $10 \mathrm{~dB}$. A $11.5-\mathrm{dB}$ SNR threshold enables the system to obtain the highest throughput when using a 8-Mbps stream. Similarly, the optimal SNR threshold for a stream of $2 \mathrm{Mbps}$ is $12.5 \mathrm{~dB}$. As discussed before, a system without considering the buffer size and buffer status will always assume a full occupancy of the buffer. In our experimental scenario, a 32-Mbps stream predominantly keeps the buffer full because the average channel capacity is around $16 \mathrm{Mbps}$. However, if we do not take offered load distribution and buffer status information into account, we would use 10-dB SNR as the adaptation threshold. As a result, there will be about 3 percent throughput degradation for the 8-Mbps stream and 5 percent degradation for the 2-Mbps stream.

In Table III, we list the different optimal thresholds for 8rate adaptation for different offered load and buffer sizes. We 
TABLE III. 8-RATE AdAPTATION THRESHOLDS

\begin{tabular}{|c|c|c|c|c|c|c|c|c|}
\hline \multirow{2}{*}{ Offered Load } & \multirow{2}{*}{ Buffer Size } & \multicolumn{7}{|c|}{ Adaptation Threshold (dB) } \\
\cline { 3 - 9 } & & $\gamma_{1}$ & $\gamma_{2}$ & $\gamma_{3}$ & $\gamma_{4}$ & $\gamma_{5}$ & $\gamma_{6}$ & $\gamma_{7}$ \\
\hline \multirow{2}{*}{$2 \mathrm{Mbps}$} & 2048 Byte & 10.0 & 11.0 & 12.0 & 14.0 & 18.0 & 20.0 & 22.5 \\
\cline { 2 - 9 } & 8192 Byte & 11.0 & 12.0 & 14.5 & 15.0 & 19.0 & 22.0 & 23.0 \\
\hline \multirow{2}{*}{$8 \mathrm{Mbps}$} & 2048 Byte & 6.0 & 8.0 & 10.0 & 13.0 & 16.0 & 20 & 22.5 \\
\cline { 2 - 9 } & 8192 Byte & 6.5 & 9.0 & 11.0 & 14.0 & 17.0 & 22.0 & 23.0 \\
\hline \multirow{2}{*}{$32 \mathrm{Mbps}$} & 2048 Byte & 4.0 & 5.5 & 8.0 & 11.5 & 14.5 & 19.0 & 22.5 \\
\cline { 2 - 9 } & 8192 Byte & 4.5 & 6.0 & 8.5 & 12.0 & 15.0 & 19.5 & 23.0 \\
\hline No Buffer/Load Information & 4.0 & 5.3 & 8.0 & 11.2 & 14.6 & 19.3 & 22.7 \\
\hline
\end{tabular}

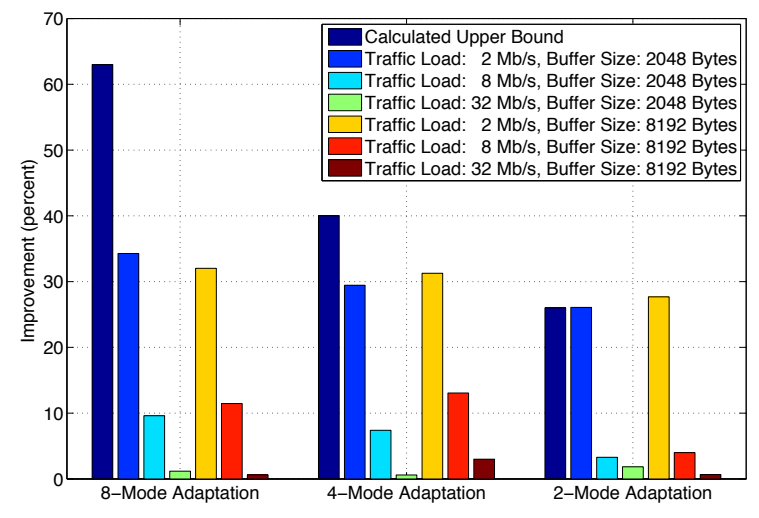

Fig. 4. Throughput improvement by exploiting offered load distribution and buffer information for different offered load and adaptation schemes.

can see that, for different offered load values, the best rate adaptation thresholds vary dramatically.

Effect of Diverse Buffer Sizes. We examined the different optimal thresholds for different buffer sizes with the same offered load. We set the offered load to $8 \mathrm{Mbps}$ in our experiments and adaptively change the transmission rate between rate 0 and rate 4 . Clearly, in Figure 3, for an 8-packet buffer, we find a different optimal adaptation threshold from the case of a 2-packet buffer. With an 8-Mbps stream, the best threshold is $12.5 \mathrm{~dB}$ for an 8 -packet buffer, while the best threshold is $11.0 \mathrm{~dB}$ for a 2-packet buffer. The intuitive explanation is that, compared to the 8-packet buffer, the 2packet buffer is more likely to overflow due to the dynamic offered load and dynamic channel capacity. In order to get a balance between the overflow loss and the channel corruption loss, the threshold should be lower in order to transmit more packets in the channel to maximize total throughput.

Improvement Evaluation. The throughput gain with buffer-information-assisted rate adaptation is clear in Figure 4. Since rate adaptation requires channel feedback from the receiver, we consider different adaptation schemes requiring a different number of feedback bits from the receiver to the transmitter. For 8-rate adaptation, we use all 8 rates listed in Table I. Rate 0, 2, 4 and 6 are used for 4-rate adaptation. Lastly, we select between rate 0 and rate 4 in 2-rate evaluation. Clearly, we can see that, the improvement increases as the offered load decreases. For a packet rate of $2 \mathrm{Mbps}$, the performance may be improved as much as 35 percent with only the statistical offered load and buffer information known. The improvement is even higher for a lower offered load than 2.0 Mbps. Moreover, the estimated upper bound is shown in that figure, which is a good indicator to estimate the actual improvement. The upper bound is about 62 percent for our current system and channel.

\section{RELATED WORK}

Rate adaptation has been studied using loss-based and channel-quality-based mechanisms. The problem of combining finite buffer and rate adaptation has also been addressed in several papers. In [10], the authors considered a constant frame slot system, in which the number of packets transmitted during one PHY frame are different for different transmission rates. Moreover, the authors formulated the system based on a Markov Chain and found some performance improvement in terms of packet loss rate. In [11], the authors analyzed the buffer-assisted rate adaptation problem with the constraint of constant total power consumption. The authors found that, in a correlated fading channel, the structure of the optimal buffer and channel adaptive transmission policies can be in sharp contrast to water-filling strategy. The author of [12] also discussed rate adaptation with transmit buffer information in the system with partial channel information at the transmitter along with no transmitter buffer information, statistical transmit buffer information and instantaneous transmit buffer information at the receiver, respectively. All the works above aim to maximize the total throughput with the constraint of maximum average transmit power, which tends to add more delay to save power.

In [14], the authors relaxed the maximum average transmit power constraint and generally analyzed the procedure of buffer-assisted rate adaptation in a constant frame slot system and studied the packet loss rate. The complexity of this algorithm is low. However, this method can not guarantee the best results, because part of the process is not optimal in terms of throughput. Moreover, they did not consider the idle time when there is no packets in the buffer. In contrast to that algorithm, we consider and analyze a variable frame slot system and jointly consider every threshold to directly find the set of thresholds to get the optimal throughput.

\section{CONCLUSION}

In this paper, we analyzed buffer-assisted packet transmission based on the IEEE 802.11 physical layer standard. Different from prior work, we used a variable frame slots model, which is practical for IEEE 802.11-related communication systems and many other systems as mentioned in Section I. Our objective is to maximize the system throughput, with a constant transmit power. We proposed the process to calculate the rate adaptation thresholds. Moreover, we derived the upper bounds of the throughput improvement by exploiting the offered load distribution and buffer status information in the rate adaptation system. We experimentally evaluated the theoretical optimal rate adaptation thresholds and showed 
substantial improvement with consideration of offered load distribution and buffer information in the system.

In future work, extension to variable packet length and multi-users scenarios could be considered. For multi-user rate adaptation, the optimization objective will be maximizing the throughput of the entire network, which requires a different strategy compared to the single user system. Also, evaluation of both power and rate adaptation is challenging but important for mobile devices in the future.

\section{REFERENCES}

[1] J. C. Bicket, "Bit-rate selection in wireless networks," M.S. Thesis, MIT, February 2005

[2] S. Wong, S. Lu, H. Yang, and V. Bharghavan, "Robust rate adaptation for 802.11 wireless networks," in ACM MobiCom, 2006.

[3] M. Lacage, M. Hossein, and T. Turletti, "IEEE 802.11 rate adaptation: A practical approach," Master's thesis, October 2004.

[4] A. Kamerman and L. Monteban, "WaveLAN II: A high-performance wireless LAN for the unlicensed band," Bell Labs Technical Journal, pp. 118-133, Summer 1997.

[5] J. Kim, S. Kim, S. Choi, and D. Qiao, "CARA: Collision-aware rate adaptation for IEEE 802.11 WLANs," in IEEE INFOCOM, 2006.

[6] B. Sadeghi, V. Kanodia, A. Sabharwal, and E. Knightly, "Opportunistic media access for multirate ad hoc networks," in ACM MobiCom, Sept. 2002.

[7] G. Holland, N. Vaidya, and P. Bahl, "A rate-adaptive MAC protocol for multi-hop wireless networks," in ACM MobiCom, Rome, Italy, July 2001.

[8] M. Vutukuru, H. Balakrishnan, and K. Jamieson, "Cross-layer wireless bit rate adaptation," in ACM SIGCOMM, 2009.

[9] D. Halperin, W. Hu, A. Sheth, and D. Wetherall, "Predictable 802.11 packet delivery from wireless channel measurements," in $A C M S I G-$ COMM, Oct. 2010

[10] H.-C. Yang and S. Sasankan, "Analysis of channel-adaptive packet transmission over fading channels with transmit buffer management," Vehicular Technology, IEEE Transactions on, vol. 57, no. 1, pp. 404 -413 , jan. 2008.

[11] A. T. Hoang and M. Motani, "Cross-layer adaptive transmission: Optimal strategies in fading channels," Communications, IEEE Transactions on, vol. 56, no. 5, pp. 799 -807, may 2008.

[12] D. Rajan, "Exploiting transmit buffer information at the receiver in block-fading channels," EURASIP Journal on Advances in Signal Processing, vol. 2009, pp. 235 - 245, 2009.

[13] J. Camp and E. Knightly, "Modulation rate adaptation in urban and vehicular environments: Cross-layer implementation and experimental evaluation," Networking, IEEE/ACM Transactions on, vol. 18, no. 6 , pp. $1949-1962$, dec. 2010.

[14] Q. Liu, S. Zhou, and G. Giannakis, "Queuing with adaptive modulation and coding over wireless links: cross-layer analysis and design," Wireless Communications, IEEE Transactions on, vol. 4, no. 3, pp. 1142 1153 , may 2005.

[15] A. Goldsmith and S.-G. Chua, "Variable-rate variable-power mqam for fading channels," Communications, IEEE Transactions on, vol. 45, no. 10, pp. $1218-1230$, oct 1997

[16] - "Adaptive coded modulation for fading channels," Communications, IEEE Transactions on, vol. 46, no. 5, pp. 595 -602, may 1998.

[17] IEEE Std 802.11-2007, 2007.

[18] D. Bertsekas and R. Gallager, Data Networks, 2nd ed. Upper Saddle River, NJ:Prentice-Hall, 1992.

[19] A. Leon-Garcia, Probability and Random Process for Electrical Engineering, 2nd ed. Addison-Wesley Publishing Company, 1994.

[20] G. L. Stuber, Principles of Mobile Communications, 2nd ed. Norwell, MA:Kluwer, 2000.

[21] H. S. Wang and N. Moayeri, "Finite-state markov channel-a useful model for radio communication channels," Vehicular Technology, IEEE Transactions on, vol. 44, no. 1, pp. $163-171$, feb 1995.
[22] J. Razavilar, K. Liu, and S. Marcus, "Jointly optimized bit-rate/delay control policy for wireless packet networks with fading channels," Communications, IEEE Transactions on, vol. 50, no. 3, pp. $484-494$, mar 2002.

[23] P. O. Murphy, "Design, implementation and characterization of a cooperative communications system," Ph.D. dissertation, Rice University, TX, USA, 2010.

[24] [Online]. Available: http://www.azimuthsystems.com 